\title{
Evolutionary Game Theory in Multi-Objective Optimization Problem
}

\author{
MAOZHU JIN \\ Business School of Sichuan University \\ Chengdu, 610065, P.R. China \\ E-mail: jinmaozhu@scu.edu.cn \\ XIA LEI * \\ National Key Laboratory of Communications, University of Electronic Science and Technology of China \\ Chengdu, 610065, P.R. China \\ E-mail: leixia@uestc.edu.cn \\ JIAN DU \\ National Key Laboratory of Communications, University of Electronic Science and Technology of China \\ Chengdu, 610065, P.R. China \\ E-mail: chiendu@uestc.edu.cn
}

\begin{abstract}
Multi-objective optimization focuses on simultaneous optimization of multiple targets. Evolutionary game theory transforms the optimization problem into game strategic problem and using adaptable dynamic game evolution process intelligently obtains the optimized strategy. The problem of multiple frequency offsets estimation in distributed multiple inputs and multiple outputs system is real-world multi-objective search and optimization problems which are naturally posed as non-linear programming problems having multiple objectives. Simulation results evidence the proposed algorithm is superior to other algorithms with more robust convergence and environmental applicability.
\end{abstract}

Keywords: Multi-Objective Optimization, Evolutionary game, Non-linear Programming Problem, Distributed multiple inputs and multiple outputs.

\section{Introduction}

\subsection{Multi-Objectives Optimization Strategies based on Evolutionary game}

Conventional optimization usually requires that objective function is continued and differentiable. Search direction of next step defined by derivative of objective function is short of simplicity and commonality. When problem becomes more complex and large scaled, search time will be expanded significantly. Evolutionary computing is a stochastic search algorithm based on natural law and its achievements have got wide attention in academic cycle which is attributed to its characteristics of adaptively and global optimization, all of which are resulted from the separation from the independence on derivative of objective function.

True to its name, evolutionary game theory appeared first in biology. Previous work by John Maynard Smith and his collaborators in biology and mathematics combined basic conception of game theory and principles of biological evolutionary optimization and in 1973 Maynard and Price put forward the central concept of "evolutionary stable strategy" which was developed

* Corresponding author 
further in Maynard Smith's (1982) influential Evolution and the Theory of Games, and these events symbolized the commence of research wave on evolutionary game theory.

Both genetic algorithm and evolutionary computing consider the biological evolving process as directed imitated object of adapted global optimization and usually are called evolutionary computing methods. The context used to interpret an evolutionarily stable strategy envisions a large population of agents who are repeatedly, randomly matched in pairs to play a game. Evolutionary game in biological model also involves biological evolutionary process, coordination evolving process of biological population in particular, so combination of evolutionary computing and game theory was a natural selection in essence.

Evolutionary game optimization method based on economic game theory maps search space of optimization problem into the combinational space of game strategies and objective function into utility function. ${ }^{1}$ In an evolutionary game, through dynamic evolutionary process of fitted individual can optimization problem be solved, each individual chooses among alternative actions or behaviors whose payoff or fitness depends on the choices of others. Over time the distribution of observed behavior in a population evolves, as fitter strategies become more prevalent.

Global convergence of evolutionary game optimization algorithms has championed its popularity in optimization area in recent years. ${ }^{2}$ One optimization problem can be represented by ${ }^{3}$ :

$$
\underset{\mathbf{x} \in D}{\arg \max } f(\mathbf{x})
$$

Where

$$
D \subseteq R^{n}
$$

is called search space.

$$
f: D \rightarrow R
$$

is objective function. Usually $\mathbf{x}=\left(x_{1}, x_{2} \ldots x_{n}\right) \in \mathbf{R}^{n}$, $x_{i} \in\left[x_{i}^{l}, x_{i}^{u}\right], i=1,2 \ldots n$ is $n$ vector. $x_{i}^{l}$ represents minimum value of $x_{i}, \quad x_{i}^{u}$ is maximum value of $x_{i}$. Set $\mathbf{x}^{*} \in D$ if $\forall \mathbf{x} \in D$, and

$$
f\left(\mathbf{x}^{*}\right) \geq f(\mathbf{x})
$$

$$
E(\xi \ln \xi) \geq(E \xi) \ln (E \xi)
$$

is the global optimum of this problem.

If there exist $\mathbf{x}^{*}$

$$
\left\{\begin{array}{l}
\mathbf{x}^{*} \in D \\
N_{\varepsilon}\left(\mathbf{x}^{*}\right)=\left\{\mathbf{x} \mid\left\|\mathbf{x}-\mathbf{x}^{*}\right\|<\varepsilon, \varepsilon>0\right\}
\end{array}\right.
$$

which satisfy (4), when

$$
\mathbf{x} \in D \cap N_{\varepsilon}\left(\mathbf{x}^{*}\right)
$$

$\mathbf{x}^{*}$ is the local optimum of this problem.

\subsection{Multi-Objective Optimization in Distributed MIMO Systems}

Multi-objective parameter optimization originated from some researching of economic balance and competition equilibrium belong to economics, in the well-known economist. Pareto's book concerning economic welfare theory, he proposed multi-objective optimization problem. To date, multi-objective optimization not only has made many significant achievements in theory, but expanded its application field increasingly. As a functional tool of solving problems from the scope of engineering technology, economic, administration, military and systems engineering, multi-objective decision foregrounds its powerful vitality gradually. 4,5 Distributed multi-input multi-output (Distributed MIMO) system is an important application area of future communication technique. It uses multiple parallel channels transmission method so that it can achieve higher transmission efficiency. After applied the distributed antenna configuration, the original singleobjective optimization problem become a typical multiobjective optimization problem because transmitting antennas or receiving antennas located in different geographic locations and using different crystal lead to different transmitting antennas to the same receiving antennas has different frequency offset.

Even a general Distributed MIMO system that transmitting antenna located in a different location, the mobile service also uses a distributed antenna. At this point, we could consider that the receiving terminal's each receiving antenna and the transmitting terminal's same transmitting antenna have a different time delay and frequency offset, and also with the transmitting terminal's different transmitting antenna. Under such

thus, 
assumptions, the time matrix offset and frequency offset matrix could extended to

$$
\begin{gathered}
\boldsymbol{\Theta}=\left[\theta_{i, j}\right]=\left[\begin{array}{cccc}
\theta_{1,1} & \theta_{1,2} & \ldots & \theta_{1, M_{T}} \\
\theta_{2,1} & \theta_{2,2} & & \theta_{2, M_{T}} \\
\vdots & & \ddots & \\
\theta_{M_{R}, 1} & \theta_{M_{R}, 2} & \ldots & \theta_{M_{R}, M_{T}}
\end{array}\right] \\
\mathbf{E}=\left[\varepsilon_{i, j}\right]=\left[\begin{array}{cccc}
\varepsilon_{1,1} & \varepsilon_{1,2} & \ldots & \varepsilon_{1, M_{T}} \\
\varepsilon_{2,1} & \varepsilon_{2,2} & & \varepsilon_{2, M_{T}} \\
\vdots & & \ddots & \\
\varepsilon_{M_{R}, 1} & \varepsilon_{M_{R}, 2} & \ldots & \varepsilon_{M_{R}, M_{T}}
\end{array}\right]
\end{gathered}
$$

Where $\theta_{i, j}$ denotes time offset between receiving antenná $i$ and receiving antenna $j, \varepsilon_{i, j}$ denotes normalized frequency offset between receiving antenna $i$ and receiving antenna $j$.

Eq. (8) and (9) shows that when transmitting antenna and receiving antenna are the distributed case, frequency estimation becomes multi-parameters estimation problem. To solve this problem, Y. Yao proposed Quasi-optimal parameter optimization strategy $^{6}$, as it essentially transfers joint multi-objective optimization to quasi-optimal single-objective optimization, its parameter estimation performance and applicability are limited.

Refs. 7 proposed joint multi-parameter optimization strategy of multi-frequency offset parameter, which took into account of the case of the same time delay. Based on traversing search optimal strategy's estimated model, respectively, through the expectation maximization (Expectation Maximum, EM) and SAGE (Space-Alternating Generalized ExpectationMaximization) algorithm to simplify multi-objective optimization strategies, experimental data show that performance of the algorithm proposed in this paper can be close to the Cramer-Rao lower bound (CRLB). It is noteworthy that these methods can achieve estimation accuracy reach to CRLB under high signal to noise ratio (SNR), but the EM algorithm's local convergence properties and sensitivity to initial conditions can make its performance deteriorating in low SNR circumstances. The proposed multi-objective optimization algorithm based on evolutionary game is superior to other algorithms with more robust convergence and applicability, especially to joint optimized strategy searching problem with multi-channel and multiparameter.

\section{System Model}

In recent years, Distributed MIMO system has attracted attention since it can counteract large-scale fading (path loss and shadow fading) and improve coverage, link quality and system capacity. The concept of Distributed MIMO system was first proposed by Saleh in 1987 in order to solve the wireless communication coverage in house $^{8}$. In this case, each transmit/receive antenna is equipped with its own oscillator; therefore, different transmit/receiver pair may have different carrier frequency offset and the performance of such systems may seriously degrade in the presence of frequency offsets due to poor synchronization. Because of this, it is of primary importance to accurately estimate these frequency offsets and compensate for them prior to performing detection. Distributed MIMO system distributes its antenna in different locations, illustrated by Fig. 1, so it is much more complicated to estimate multi-frequency offsets, as the existing algorithms for the single-input, single-output (SISO) system cannot be applied directly to Distributed MIMO systems.

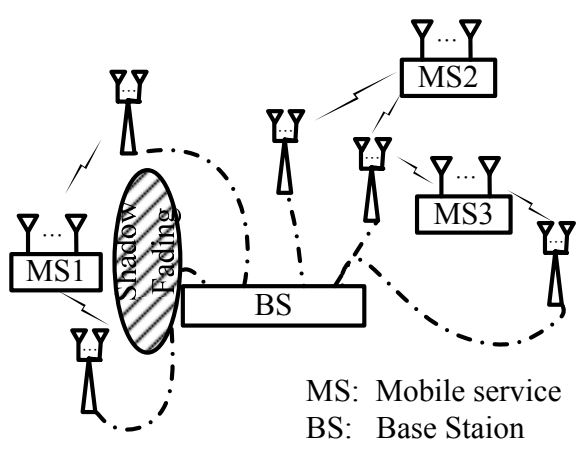

Fig.1 Schematic diagram of distributed MIMO systems

In distributed MIMO system, system distributes its antennas of base station in different locations and these antennas are connected to a central processor through fiber or coaxial cable. Thus, each antenna sends received information of mobile service to central processor and in the meantime central processor also sends information to mobile service through multiple antennas of base station using fiber or coaxial cable. Considering in a flat-fading MIMO system, the $k$ th receiving antenna receive information at the time of $n$ : 


$$
y_{k}(n)=\sum_{l=1}^{M_{T}} h_{k, l} e^{j \varepsilon_{k, l} n} s_{l}(n)+n_{k}(n), n=1,2, \ldots, N
$$

where training series $\left\{s_{l}(n)\right\}_{n=1}^{N}$ is known. Define

$$
S_{\varepsilon_{k}}=\left[\begin{array}{cccc}
s_{1}(1) e^{j \varepsilon_{k, 1}} & s_{2}(1) e^{j \varepsilon_{k, 2}} & \cdots & s_{M_{T}}(1) e^{j \varepsilon_{k, N_{T}}} \\
s_{1}(2) e^{j 2 \varepsilon_{k, 1}} & s_{2}(2) e^{j 2 \varepsilon_{k, 2}} & \cdots & s_{M_{T}}(2) e^{j 2 \varepsilon_{k, N_{T}}} \\
\cdots & \cdots & \cdots & \cdots \\
s_{1}(N) e^{j N \varepsilon_{k, 1}} & s_{2}(N) e^{j N \varepsilon_{k, 2}} & \cdots & s_{M_{T}}(N) e^{j N \varepsilon_{k, N_{T}}}
\end{array}\right]
$$

Then maximum likelihood estimation of communication channel and frequency offset can get from minimizing logarithm likelihood function.

$$
\Lambda=\sum_{k=1}^{M_{R}}\left\|y_{k}-S_{\varepsilon_{k}} h_{k}\right\|^{2}
$$

That is equivalent to minimize

$$
\Lambda_{k}=\left\|y_{k}-S_{\varepsilon_{k}} h_{k}\right\|^{2}
$$

Fixing $w_{k}$, we can get

$$
\hat{h}_{k}=\left(S_{\varepsilon_{k}}^{H} S_{\varepsilon_{k}}\right)^{-1} S_{\varepsilon_{k}}^{H} y_{k}
$$

Then multi-frequency offset estimation problem can be transformed to multi-objective optimization of

$$
\hat{\varepsilon}_{k}=\arg \max _{\varepsilon_{k}} y_{k}^{H} S_{\varepsilon_{k}}\left(S_{\varepsilon_{k}}^{H} S_{\varepsilon_{k}}\right)^{-1} S_{\varepsilon_{k}}^{H} y_{k}
$$

Eq. (15) indicates that multi-frequency offset estimation problem is the special case of multi-objective optimization. Traversal search methods are too complex to implement, so in recent years, this problem has got a wide attention. ${ }^{9-12}$ In which Refs. 7 improved EM algorithm to accelerating algorithm ECM and simplified multi-dimensional search effort to estimate multifrequency offset using SAGE, the mean square error of estimation approached CRLB.

\section{1. $E M$}

EM is an iterative optimization method to estimate some unknown parameters, given measurement data. ${ }^{13-15}$ However, we are not given some "hidden" nuisance variables, which need to be integrated out. The intuition behind EM is an old one: alternate between estimating the unknowns and the hidden variables. This idea has been around for a long time. One of the most insightful explanations of EM, that provides a deeper understanding of its operation than the intuition of alternating between variables, is in terms of lower bound maximization (Neal and Hinton, 1998; Minka, 1998).

The derivation of EM algorithm depends on an assumption which is call complete data space $\mathrm{x}$. there is mapping relationship $y=g(x)$ between observed stochastic variable $\mathrm{y}$ in incomplete data space and $\mathrm{x}$. Function $g$ is a many-to-one transformation. In the $m$ th iteration, EM algorithm first computes expectation. This is called E-step (Expectation Step):

$$
Q\left(\theta \mid \hat{\theta}^{[m]}\right)=E\left\{\log f(x \mid \theta) \mid y, \hat{\theta}^{[m]}\right\}
$$

The second step is called M-step (Maximization Step) which updates parameter vector

$$
\hat{\theta}^{[m+1]}=\arg \max _{\theta} Q\left(\theta \mid \hat{\theta}^{[m]}\right)
$$

The representation of EM is relatively complex, but it is really amazing to use simple multi-step iteration to get maximum likelihood estimation. In this derivation, the E-step can be interpreted as constructing a local lowerbound to the posterior distribution, whereas the M-step optimizes the bound, thereby improving the estimate for the unknowns. Its physical meaning is obvious that $\mathrm{m}$ step of algorithm guarantees the incremental value of likelihood function in each step. It can be proved that posterior value of likelihood function is larger or equal to the value before iteration. Hence, through multi-step iteration, EM algorithm can converge to local optimum neighbouring the initial value.

Whether or not EM algorithm can search out global maximum depends on initial value $\hat{\theta}^{[0]}$. Its convergence rate is inversely proportional to the conditional Fisher information matrix of $x, y$ [Appendix A]. When dimensions of complete data space is large, convergence rate will be low, so the application of this algorithm is sensitive to its applied environment.

\subsubsection{ECM}

In some case, when M-step EM algorithm is too complex, ECM (Expectation Conditional Maximization) algorithm can simplify its computing ${ }^{16}$. If parameter vector $\theta$ can be divided into $\mathrm{M}$ group including $\theta_{l}, l=1,2 \ldots M$, then the $m$ th iteration of EM algorithm can be divided into M substeps, in which $\theta_{l}$ 
is updated at the $l$ th substep. At this time, $\theta_{v}, v \neq l$ is fixed as the last updated value.

The lth step includes:

(i) Search :

$$
\hat{\theta}_{l}^{[m+1]}=\left.\arg \max _{\theta_{l}} Q\left(\theta \mid \hat{\theta}^{[m]}\right)\right|_{\theta_{v}=\hat{\theta}_{v}^{[m]}, v \neq l}
$$

(ii) Updated:

$$
\hat{\theta}_{l}^{[m]}=\hat{\theta}_{l}^{[m+1]}
$$

In the backdrop of Distributed MIMO system, ECM algorithm is applied to solve multi-parameter optimization. First, the $l$ Transmitting antenna training series and frequency offset are defined by

$$
\begin{gathered}
s_{l}=\left[\begin{array}{llll}
s_{l}(1) & s_{l}(2) & \cdots & s_{l}(N)
\end{array}\right]^{T} \\
e_{k, l}=\left[\begin{array}{llll}
e^{j w_{k, l}} & e^{j 2 w_{k, l}} & \cdots & e^{j N w_{k, l}}
\end{array}\right]^{T}
\end{gathered}
$$

The receiving signal of the $k$ th receiving antenna is

$$
y_{k}=\sum_{l=1}^{N_{T}}\left(s_{l} \odot e_{k, l}\right) h_{k, l}+n_{k}
$$

In EM algorithm, receiving signal $y_{k}$ constructs incomplete space. Solve for parameter is

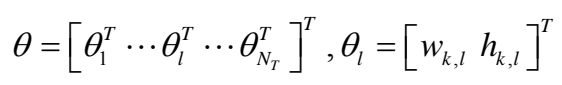

Define complete data space

$$
z_{k}=\left[z_{k, 1} z_{k, 2} \cdots z_{k, N_{T}}\right]^{T}
$$

where

$$
z_{k, l} \triangleq\left(s_{l} \odot e_{k, l}\right) h_{k, l}+n_{k, l} \quad, \quad l=1,2, \cdots, N_{T}
$$

Thus, relationship between $z_{l}$ and $y_{k}$ is

$$
\sum_{l=1}^{N_{T}} z_{k, l}=y_{k}
$$

(i) E-step: computing expectation

$$
Q\left(\theta \mid \hat{\theta}^{[m]}\right) \triangleq E\left\{\log f\left(z_{k} \mid \theta\right) \mid y_{k}, \hat{\theta}^{[m]}\right\}
$$

$n_{k, l}$ is statistically independent, so probability density function of $z_{k}$ to $\theta$ is

$$
\begin{aligned}
f\left(z_{k} \mid \theta\right) & =\prod_{l=1}^{N_{T}} f\left(z_{k, l} \mid \theta_{l}\right) \\
& =\prod_{l=1}^{N_{T}} \frac{1}{\left(\pi \beta_{l} \sigma^{2}\right)^{N}} \exp \left\{-\frac{\left\|z_{k, l}-\left(s_{l} \odot e_{k, l}\right) h_{k, l}\right\|^{2}}{\beta_{l} \sigma^{2}}\right\}
\end{aligned}
$$

Suppose

$$
\beta_{l}=\frac{1}{N_{T}}
$$

substitute Eq. (28) into Eq. (27), get

$$
Q\left(\theta \mid \hat{\theta}^{[m]}\right)=C_{2}-\sum_{l=1}^{N_{T}} \frac{1}{\beta_{l} \sigma^{2}}\left\|\hat{z}_{k, l}^{[m]}-\left(s_{l} \odot e_{k, l}\right) h_{k, l}\right\|^{2}
$$

where

$$
\hat{z}_{k, l}^{[m]} \triangleq E\left\{z_{k, l} \mid y_{k}, \hat{\theta}^{[m]}\right\}
$$

Suppose $h_{k, l}$ is the independent and identically distributed (i.i.d.) complex Gaussian variables with zero-mean and variance 1 . And $z_{k}$, and $y_{k}$ are joint Gaussian distributed, so

$$
\begin{aligned}
\hat{z}_{k, l}^{[m]}= & \left(s_{l} \odot \hat{e}_{k, l}^{[m]}\right) \hat{h}_{k, l}^{[m]} \\
& +\beta_{l}\left(y_{k}-\sum_{v=1}^{N_{T}}\left(s_{v} \odot \hat{e}_{k, v}^{[m]}\right) \hat{h}_{k, v}^{[m]}\right)
\end{aligned}
$$

where

$$
\hat{e}_{k, v}^{[m]}=\left[e^{j \hat{w}_{k, v}^{[m]}} e^{j 2 \hat{\hat{k}}_{k, \nu}^{[m]}} \cdots e^{j N \hat{w}_{k, v}^{[m]}}\right]^{T}
$$

(ii) M-step: maximize solution

$$
\hat{\theta}^{[m+1]}=\arg \min _{\theta} \sum_{l=1}^{N_{T}}\left\|\hat{\mathbb{Z}}_{k, l}^{[m]}-\left(s_{l} \odot e_{k, l}\right) h_{k, l}\right\|^{2}
$$

Eq. (34) can be divided into $N_{T}$ independent process

$$
\hat{\theta}_{l}^{[m+1]}=\arg \min _{\theta_{l}}\left\|\hat{z}_{k, l}^{[m]}-\left(s_{l} \odot e_{k, l}\right) h_{k, l}\right\|^{2}
$$

Different from synchronously updating solve for parameter in EM algorithm, ECM algorithm divides Eq. (35) into two steps. In the first step, update one of parameters $\left(w_{k, l}, h_{k, l}\right)$, and at the same time keep other parameter as the latest updating value. $\hat{\theta}_{l}^{[m+c / 2]}$ represents estimation of $\theta_{l}$ at c-step in the $m$ th iteration 
$(c=1,2)$. So $M$-step of EM algorithm includes two substeps:

(i) Step 1:

Set

$$
h_{k, l}=\hat{h}_{k, l}^{[m]}
$$

update $w_{k, l}$. That is, computing

$$
\hat{\theta}_{l}^{[m+1 / 2]}=\left[\hat{w}_{k, l}^{[m+1]} \hat{h}_{k, l}^{[m]}\right]^{T}
$$

and

$$
\begin{array}{r}
\hat{w}_{k, l}^{[m+1]}=\arg \max _{w_{k, l}} \sum_{t=1}^{N} \mathfrak{R}\left\{\left(\hat{z}_{k, l}^{[m]}(t)\right)^{*} s_{l}(t) \hat{h}_{k, l}^{[m]} e^{j w_{k, l}}\right\} \\
, t=1,2, \ldots, N
\end{array}
$$

$e^{j w_{k, l} t}$ is expanded in Taylor series of second order at $\hat{w}_{k, l}^{[m]}$, get

$$
\begin{aligned}
e^{j w_{k, l} t} \approx & e^{j \hat{w}_{k, l}^{[m]} t}+\left(w_{k, l}-\hat{w}_{k, l}^{[m]}\right)(j t) e^{j \hat{w}_{k, l}^{[m]} t} \\
& +\frac{1}{2}\left(w_{k, l}-\hat{w}_{k, l}^{[m]}\right)^{2}(j t)^{2} e^{j \hat{w}_{k, l}^{[m]} t}
\end{aligned}
$$

Substitute Eq. (39) into Eq. (38) and differentiate $\{\cdot\}$ to $w_{k, l}$ and let the result be equal 0 , the updated $\hat{w}_{k, l}^{[m+1]}$ is

$$
\hat{w}_{k, l}^{[m+1]}=\hat{w}_{k, l}^{[m]}-\frac{\sum_{t=1}^{N} t \mathfrak{I}\left\{\left(\hat{z}_{k, l}^{[m]}(t)\right)^{*} s_{l}(t) \hat{h}_{k, l}^{[m]} e^{j \hat{w}_{k, l}^{[m]} t}\right\}}{\sum_{t=1}^{N} t^{2} \mathfrak{R}\left\{\left(\hat{z}_{k, l}^{[m]}(t)\right)^{*} s_{l}(t) \hat{h}_{k, l}^{[m]} e^{j \hat{w}_{k, l}^{[m]} t}\right\}}
$$

Simulation results indicate the relevant function is convex.

(ii) Step 2:

Fix $w_{k, l}$, update $h_{k, l}$ to $\hat{h}_{k, l}^{[m+1]}$. That is, computing

$$
\hat{\theta}_{l}^{[m+1]}=\left[\hat{w}_{k, l}^{[m+1]} \hat{h}_{k, l}^{[m+1]}\right]^{T}
$$

and

$$
\hat{h}_{k, l}^{[m+1]}=\frac{1}{\sum_{t=1}^{N}\left|s_{l}(t)\right|^{2}} \sum_{t=1}^{N} \frac{\hat{z}_{k, l}^{[m]}(t) s_{l}^{*}(t)}{e^{j w_{k, l}^{[m+1]} t}}
$$

Change the value of $k$ and repeat above-mentioned algorithm in $N_{R}$ times, estimation values of all receiving antennas can be computed.

\subsubsection{SAGE}

As convergence rate of EM algorithm is relatively low, SAGE (Space-Alternating Generalized Expectation-
Maximization) ${ }^{\mathbf{1 7}}$ algorithm is proposed to fill up this inefficiency. The methodology of SAGE is the division of parameter vector into smaller groups and updates them. When a small group is updated, others' parameters remain the same. In SAGE algorithm, every group of parameters constructs a hidden data space but not a big complete data space. Suppose $\theta_{S}$ represents the vector including $\mathrm{S}$ parameters in the vector $\theta, \theta_{\tilde{s}}$ represents the vector including remaining parameters, then $x^{S}$ is the accommodation hidden data space. In SAGE algorithm, update of $\theta_{S}$ includes two steps:

(i) E-step:

Determine conditional logarithm likelihood expectation

$$
Q_{S}\left(\theta_{S} \mid \hat{\theta}^{[m]}\right)=E\left\{\log f\left(x^{S} \mid \theta_{S}, \hat{\theta}_{\tilde{S}}^{[m]}\right) \mid y, \hat{\theta}^{[m]}\right\}
$$

(ii) M-step:

Search maximum

$$
\hat{\theta}_{S}^{[m+1]}=\arg \max _{\theta_{S}} Q_{S}\left(\theta_{S} \mid \hat{\theta}^{[m]}\right)
$$

In the backdrop of distributed MIMO system, SAGE algorithm is also applied to solve multi-parameter optimization.

Divide solve for parameter into $N_{T}$ groups of

$$
\theta_{l}, l=1,2, \ldots, N_{T}
$$

when one group is updated, others remain the same. Hidden data space of $\theta_{l}$ can be defined by

$$
x_{k, l} \triangleq\left(s_{l} \odot e_{k, l}\right) h_{k, l}+n
$$

Where all $n$ are correlated to hidden data space so that Fisher information matrix of hidden data space can be reduced to improve convergence rate.

Updating process of $\theta_{l}$ in the $m$ th iteration is described in following, also including $E$-step and $M$-step. In the $m$ th iteration, these two steps will be executed $N_{T}$ times in order to update all $\theta_{l}$.

(i) E-step:

With the constraint conditions of $y_{k}, \hat{\theta}^{[m]}$, keep $\theta_{v}$, $v \neq l$ constant, compute the expectation of logarithm likelihood function of hidden data space,

$$
Q\left(\theta_{l} \mid \hat{\theta}^{[m]}\right)=E\left\{\log f\left(x_{k, l} \mid \theta_{l},\left\{\hat{\theta}_{v}^{[m]}\right\}_{v \neq l}\right) \mid y_{k}, \hat{\theta}^{[m]}\right\}
$$

then 


$$
\begin{aligned}
f\left(x_{k, l} \mid \theta_{l},\left\{\hat{\theta}_{v}^{[m]}\right\}_{v \neq l}\right) & =f\left(x_{k, l} \mid \theta_{l}\right) \\
& =\frac{1}{\left(\pi \sigma^{2}\right)^{N}} \exp \left\{-\frac{\left\|x_{k, l}-\left(s_{l} \odot e_{k, l}\right) h_{k, l}\right\|^{2}}{\sigma^{2}}\right\}
\end{aligned}
$$

Substitute Eq. (48) into Eq. (47),

$$
Q\left(\theta_{l} \mid \hat{\theta}^{[m]}\right)=C_{4}-\frac{1}{\sigma^{2}}\left\|\hat{x}_{k, l}^{[m]}-\left(s_{l} \odot e_{k, l}\right) h_{k, l}\right\|^{2}
$$

Where

$$
\begin{aligned}
\hat{x}_{k, l}^{[m]} & \triangleq E\left\{x_{k, l} \mid y_{k}, \hat{\theta}^{[m]}\right\} \\
& =y_{k}-\sum_{v=1, v \neq l}^{N_{T}}\left(s_{v} \odot \hat{e}_{k, v}^{[m]}\right) \hat{h}_{k, v}^{[m]}
\end{aligned}
$$

(ii) M-step: update $\hat{\theta}_{l}^{[m+1]}$

$$
\begin{aligned}
\hat{\theta}_{l}^{[m+1]} & =\arg \max _{\theta_{l}} Q\left(\theta_{l} \mid \hat{\theta}_{l}^{[m]}\right) \\
& =\arg \min _{\theta_{l}}\left\|\hat{x}_{k, l}^{[m]}-\left(s_{l} \odot e_{k, l}\right) h_{k, l}\right\|^{2}
\end{aligned}
$$

Same as ECM algorithm, Eq. (51) can be divided into two substeps:

(i) Step 1: get $\hat{w}_{k, l}^{[m+1]}$

$$
\begin{aligned}
& \hat{w}_{k, l}^{[m+1]}=\hat{w}_{k, l}^{[m]}-\frac{\sum_{t=1}^{N} t \mathfrak{J}\left\{\left(\hat{x}_{k, l}^{[m]}(t)\right)^{*} s_{l}(t) \hat{h}_{k, l}^{[m]} e^{\left.j \hat{w}_{k, l}^{[m]}\right\}}\right.}{\sum_{t=1}^{N} t^{2} \mathfrak{R}\left\{\left(\hat{x}_{k, l}^{[m]}(t)\right)^{*} s_{l}(t) \hat{h}_{k, l}^{[m]} e^{j \hat{w}_{k, l}^{[m]} t}\right\}} \\
& \text { (ii) Step 2: get } \hat{h}_{k, l}^{[m+1]}
\end{aligned}
$$

$$
\hat{h}_{k, l}^{[m+1]}=\frac{1}{\sum_{t=1}^{N}\left|s_{l}(t)\right|^{2}} \sum_{t=1}^{N} \frac{\hat{x}_{k, l}^{[m]}(t) s_{l}^{*}(t)}{e^{j \hat{w}_{k, l}^{[m+1]} t}}
$$

SAGE-ECM algorithm is the combination of ECM and SAGE which also applies to each receiving antenna in order to get the estimation value of receiving information.

Maximum likelihood estimation aims to get global optimum strategy. However, both EM and other accelerating algorithms are local optimum algorithms. 6 can provide the initial value (Appendix B). But only if this value is approach the global optimum, the iteration process of algorithms can get relatively exact estimation. Since performance of EM algorithm is very sensitive to the initial value, the result of algorithm is prone to get local optimum. At the same time, because estimation range is inversely proportional to length of training series in frequency offset estimation methods based on relevant frequency offsets, estimation range of both EM and other accelerating algorithms are limited to the range of initial value estimation. The applicability of algorithms is limited too.

\subsection{Evolutionary game}

This paper propose a frequency offset estimation method based on evolutionary game, which normalized estimation range can reach the maximum range ${ }^{3}$, and mean square error of estimation reaches CRLB.

The following is a case of evolutionary game optimization method. According to Refs. 18, a noncooperative game cab be formulated by

$$
G=[I, S, U]
$$

Where, $I=\left\{1,2, \ldots, n_{I}\right\}$ represent individual number of game players, $S$ is strategy set of game players. $U$ is the utility function of game. Every component $x_{i}$, $i=1,2, \ldots n$, of variable $\mathbf{x}$ is corresponding to a strategy of game players, that is, $I=\left\{1,2, \ldots, n_{I}\right\}$. Strategy set of a game player is $S_{i}=\left[x_{i}^{l}, x_{i}^{u}\right], \quad i \in I$. Every component $x_{i}, \quad i=1,2, \ldots n$, of variable $\mathbf{x}$ is mapped to a game play in the game through $\varphi$, the utility function can be represented by

$$
u(\mathbf{s}) \equiv f\left(\varphi^{-1}(\mathbf{s})\right)=f(\mathbf{x})
$$

Theorem 1: the global optimization solution $\mathbf{x}^{*}$ of Eq. (1) reference goes here corresponding to strategy set $\mathbf{s}^{*}$ is a Pareto-optimal equilibrium point of game $G$.

Proof:

Because

$$
u_{i}(\mathbf{s}) \equiv f(\mathbf{x}), i \in I
$$

and

$$
\forall \mathbf{x} \in D, f\left(\mathbf{x}^{*}\right) \geq f(\mathbf{x})
$$

we have

$$
\forall \mathbf{s} \in S, u_{i}\left(\mathbf{s}^{*}\right) \geq u_{i}(\mathbf{s}), \forall i \in I
$$

According to de definition of Nash equilibrium, this strategy set is the Nash equilibrium point. And because under such strategy set utility of each player reach maximum, such equilibrium status is Pareto-optimal. Evolutionary process chooses the initial strategy set $\mathbf{s}^{0}$ stochastically, and through many iterations of sequential optimization response can set reach a dynamic "stable" 
state, to which artificially impose a stochastic interference that represents sudden noise, therefore, the "stable" state is disrupted and through sequential optimization response the set reaches another "stable" state. Repeat this process until the preset stopping criteria is satisfied.

\section{Definition1 set}

$$
G=[I, S, U]
$$

and

$$
S_{-i}=\prod S_{k}, k=1,2, \ldots, n, k \neq i
$$

if

$$
B_{i}\left(\mathbf{s}_{-i}\right)=\left\{s_{i}^{*} \in S_{i}: u_{i}\left(s_{i}^{*}, \mathbf{s}_{-i}\right)>u_{i}\left(s^{(i)}, \mathbf{s}_{-i}\right), \forall s^{(i)} \in S_{i}\right\}
$$

Then

$$
B_{i}: S_{-i} \rightarrow S_{i}
$$

which is called the best-response correspondence for player $i$

Under certain strategic set, if the strategies of other players remain unchanged, a player chooses the strategy to maximize its utility. This is the optimum response of the player in such strategic set. Start from certain strategic set $\mathbf{s}$, the dynamic process of each player choosing their optimum response alternatively in sequence is called optimum response dynamics.

Definition of interference operator in evolutionary process: to $i^{\text {th }}$ component $s_{i}$ of $\mathbf{s}$, that is, the strategy of the player, if random number $\operatorname{rand}(0,1)$ is lower than given interference probability $p_{d}$, then the player will randomly choose one strategy in the strategic set to replace such component, otherwise, the strategy remain unchanged. Repeat such selection method to each strategy in the strategic set, then we have strategic set s' after interference.

Another problem is how the player searches its own optimum response strategy. Suppose that parameter range of $x_{i}$, that is corresponding component of strategic set of player $i$ is $\left[x_{i}^{l}, x_{i}^{u}\right]$. Because strategies of other players remain constant, this problem is converted to a single variable optimization problem, so a simple stochastic searching process is applied to obtain the approximation of optimum response strategy. In this process, new strategy $x_{i}$ is produced by mutation, that is

$$
x_{i}^{t+1}=x_{i}^{t}+N\left(0, \sigma^{2}(t)\right), t=1,2, \ldots C
$$

Where $N\left(0, \sigma^{2}(t)\right)$ is independent Gaussian random variable with zero mean and variance $\sigma^{2}(t), C$ is preset searching number. Evolutionary rule of $\sigma$ is

$$
\sigma(t+1)=\alpha \sigma(t), 0<\alpha<1
$$

If variant value of $x_{i}$ exceeds the range of the parameter, then take one point in its range to replace it. In this search process, the strategy with maximum utility will be regarded as approximation of optimum response strategy of the player.

As for $k^{\text {th }}$ receiving antenna, $\boldsymbol{\varepsilon}_{k}=\left[\varepsilon_{k, 1} \ldots \varepsilon_{k, M_{T}}\right]$ has $n_{I}=M_{T}$ frequency offset, that is $M_{T}$ players. According to rule of maximum likelihood estimation, the game utility function can be expressed by

$$
u(\boldsymbol{\varepsilon})=y_{k}^{H} S_{\varepsilon}\left(S_{\varepsilon}^{H} S_{\varepsilon}\right)^{-1} S_{\varepsilon}^{H} y_{k}
$$

Strategic set of game players is

$$
\boldsymbol{\varepsilon}=\prod \varepsilon_{k}, k=1,2, \ldots, M_{T}
$$

where $\varepsilon_{k} \in 2 \pi(-0.5,0.5)$. Multi-frequency offset estimation can be transformed to the searching problem off maximum utility function $u(\varepsilon)$ of multiple players in given range.

In accordance with previous analysis, game structure of distributed frequency offset estimation is $G=[I, \boldsymbol{\varepsilon}, u]$, $I=\left\{1,2, \ldots, M_{T}\right\}$, where Pareto-optimal equilibrium point of game can be obtained by optimum response.

The optimum response is when a player $\omega_{i}$ has a chance to rectify its strategy, the value of new strategy will satisfy

$$
\varepsilon_{i}^{\prime} \in \arg \max _{s_{i} \in S} u_{i}\left(\varepsilon_{i}^{\prime}, \varepsilon_{-i}\right)
$$

Start from initial value combination of certain game player, the dynamic process of each player choosing their optimum response alternatively in sequence is called optimum response dynamics. When all game players adopt the same value combination after Mth iterations, this is called steady-state. According to literature 1, any steady-state of dynamic optimum response must be Nash equilibrium. This paper applies similar evolutionary optimization algorithm to solve it. $\omega_{i}^{t+1}$, new value of game player in this process will be produced by

$$
\varepsilon_{i}^{p+1}=\varepsilon_{i}^{p}+N\left(0, \sigma^{2}(p)\right), p=1,2, \ldots P
$$


Where $N$ is independent Gaussian random variable with zero mean and variance $\sigma^{2}(p), P$ is preset searching number. Evolutionary rule of $\sigma$ is ${ }^{1}$

$$
\sigma(p+1)=\alpha \cdot \sigma(p), \alpha=0.5, \sigma(1)=1
$$

If variant value of $\omega_{i}$ exceeds the range

$$
\boldsymbol{\varepsilon}=\prod_{i=1}^{N_{T}}\left[\varepsilon_{k, i}^{l}, \varepsilon_{k, i}^{u}\right]
$$

then take one point in its range to replace it.

Since normalized frequency offset $\varepsilon_{k}$ is continuously distributed in the range of $2 \pi(-0.5,0.5)$ and utility function Eq. (65) is multi-extremum function, different steady-states are resulted from different initial value. In the frequency offset searching process, when reaching the steady-state, the process of leaping out of current steady-state and searching for next steady-state is needed. Comparing the values of different steady-states, different game players can reach Pareto-optimal equilibrium point of game. According to literature 1, we can avoid the local optimization. We generate a random number which is uniform distribution on the interval $[0$, 1] for any game player, if the random number is lower than given interference probability $p_{d}$, then the player will randomly choose one strategy in the strategic set to replace current value, otherwise, stay unchanged.

Above mentioned analysis indicates that searching for Pareto-optimal equilibrium game point of multifrequency offset optimized strategy is in essence the process of $T$ times of searching different steady-state and $P$ rounds of searching optimum response.

In the real systems, due to complexity restrictions, the number of $P$ and $T$ must be limited that resulted in approximation value. If range of variables

$$
\boldsymbol{\omega}=\prod \omega_{k}, \quad k=1,2, \ldots, N_{T}
$$

is larger, the range of $P$ and $T$ needs even larger. Algorithm for the multi-frequency offset estimation based on the evolutionary game is summarized in the Table 1.

\section{Simulation Results and Analysis}

In this section, we investigate the MSE performance of our proposed algorithm in a Distributed MIMO in flat fading environment, mainly for the number of transmitter antennas $N_{T}=2$. The training sequence portion is with length of $N=32$. In the classic training sequence used in Yao's method is taken from a row of a Hadamard matrix with length $N=32$. It consists of length of $P=4$ correlator, which controls the estimation range of the frequency offset in 67 . Relevant simulation parameters are listed in table 2 .

Fig. 2 illustrates the mean square error of frequency offset estimation between sending antenna 1 and receiving antenna 1 . With increase of relevant length from 4 to 8 to 16 , the results indicate that estimation performance is improved. But according to 6 , the range of frequency offset will be reduced with the increase of relevant length.

The performance this algorithm is approaching estimation lower limit, but with the increase of SNR the interference among antennas is intensified, the algorithm proposed by Yao yield mean square error platform of estimator. This indicates that this kind of algorithm is single objective optimization in essence and does not take advantage of the strength of multiobjective joint optimization, so its application is sensitive to the environment and limited.

EM algorithm is a transversal searching method based on multi-objective joint optimization which increases its adaptability and obtains the relatively ideal solution to the problem that with the increase of SNR estimation mean square error platform exists. But the algorithm still uses relevant estimation as the initial value and local convergence, so range of estimation is limited and under low SNR the algorithm is hard to converge to the global optimum. The paper proposes the algorithm based on evolutionary game overcomes the problems that previous algorithms encounter. The following is the comparison made through numerical simulation. Performance of all algorithms is illustrated and relevant explanation is given. Relevant simulation parameters are listed in table 3.

Fig. 3 illustrates that using initial values based on Yao's method, SAGE-ECM solves the problem of interference among antennas. When SNR is higher than $10 \mathrm{~dB}$, estimation performance reaches CRLB. But when SNR is low, it is hard to converge to global optimum whose performance is even lower than that using single objective optimization method.

Fig. 4 explains this phenomenon. Analyzing the objective function of multi-objective optimization at $8 \mathrm{~dB}$, it is found that when SNR is low, there are multiple local optimum of objective function and using 
initial values based on Yao's method, estimation error is relatively big.

Table1. Summary of Estimation for the $k^{\text {th }}$ Receivers

\section{Initialization}

Random choose initial $\boldsymbol{\varepsilon}^{0}$ from the set $2 \pi[-0.5,0.5]$

2. $T$ rounds best-response correspondence

$$
\begin{aligned}
& \text { for } t=0,1 \ldots T \\
& p_{d}=1 / t \\
& \text { for } m=1 \ldots M_{R} \\
& \quad \text { for } i=1,2, \ldots M_{T}
\end{aligned}
$$

Compute the approximate bestresponse correspondence

in $P$ rounds.

$\varepsilon(t)_{i}^{\prime} \in \arg \max u_{i}\left(\varepsilon^{p}(t)_{i}\right)$

$$
, p=1,2 \ldots P
$$

end

$$
\text { if } u\left(\varepsilon(m)^{\prime}\right)>u(\varepsilon(m))
$$

$$
\boldsymbol{\varepsilon}(m+1)=\boldsymbol{\varepsilon}(m)^{\prime}
$$

else

$$
\boldsymbol{\varepsilon}(m+1)=\boldsymbol{\varepsilon}(m)
$$

end

$$
\text { end }
$$

if rand $<p_{d}$

$\varepsilon(t+1):$ choose one form $2 \pi[-0.5,0.5]$ randomly end

end

Table 2. Simulation parameters of quasi-optimal multiparameter optimization

\begin{tabular}{c|c}
\hline$N_{T}$ & 2 \\
\hline$N_{R}$ & 2 \\
\hline$Q$ & $4,8 、 16$ \\
\hline$P$ & $2 \pi\left[\begin{array}{l}0.01,0.015 \\
0.02,0.025\end{array}\right]$ \\
\hline
\end{tabular}

Because ECM algorithm increases the value of the objective function through multiple iterations, the final parameter estimation can exactly obtain the maximum of objective function. So ECM is local optimization algorithm which converges to the neighboring point of initial value. In result, ECM algorithm converges to a local optimum instead of global optimum which creates a relatively big error, so estimation accuracy is worse than initial value.

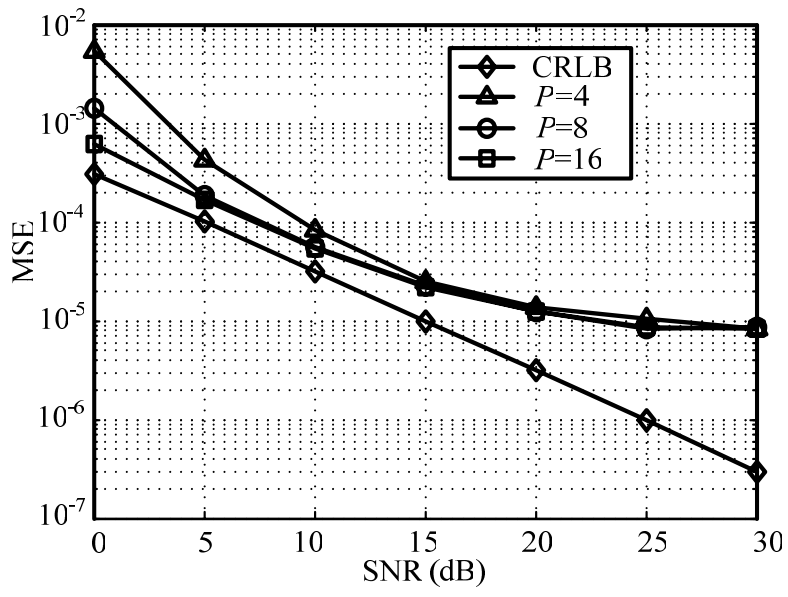

Fig. 2. Performance of quasi-optimal multi-parameter optimization method

Table 3 simulation condition for multi-frequency offset estimation

\begin{tabular}{c|c}
\hline$Q$ & 32 \\
\hline$P$ & 8 \\
\hline$\left[\omega_{1,1}, \omega_{1,2}\right]$ & $2 \pi[0.01,0.015]$ \\
\hline $\begin{array}{c}\text { Parameters for proposed } \\
\text { method }\end{array}$ & $\left\{\begin{array}{l}T=80 \\
P=7\end{array}\right.$ \\
\hline
\end{tabular}

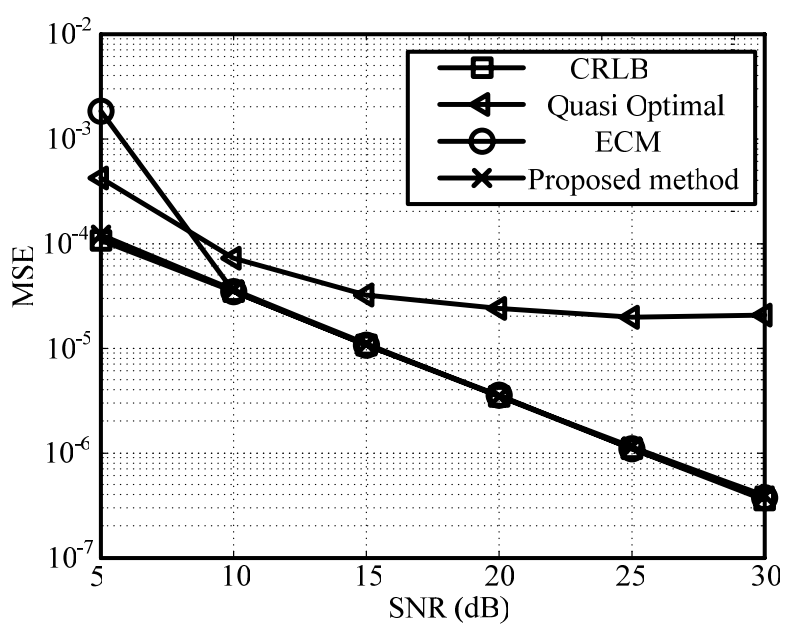

Fig. 3. Performance of different optimization methods 
The proposed algorithm based on evolutionary game avoids the occurrence of this situation. Because evolutionary game algorithm is a global optimization algorithm, when SNR is higher than some level, this method can complete optimization of multi-frequency offset parameters with accuracy of approaching CRLB.

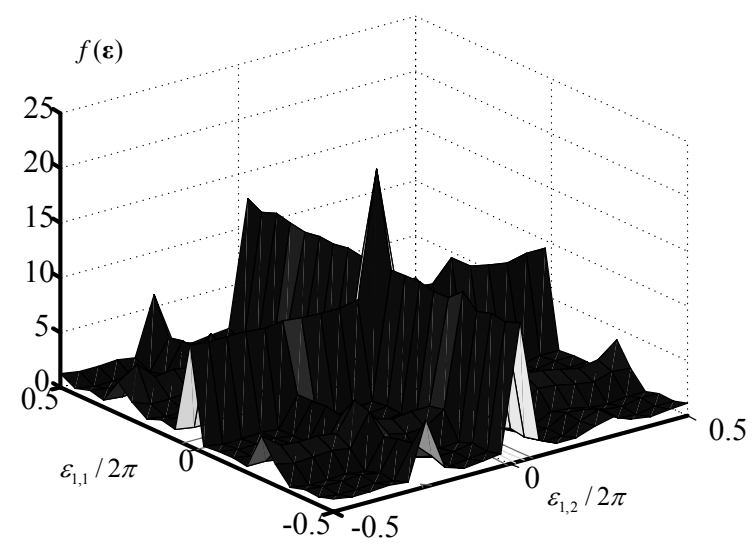

Fig. 4. Objective function value of Multi-objective optimization

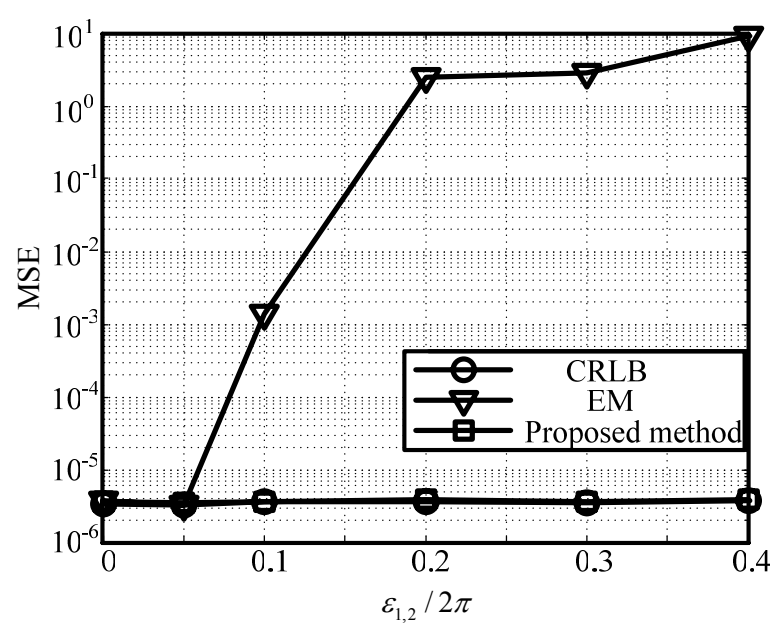

Fig. 5 Estimation performance of different method

Fig. 5 is the comparison of estimation range of several estimation algorithms which represents the applicability of the method. Estimation range of Yao's method is single objective optimization in essence, so estimation range is small. The local convergence makes EM algorithm also encounter the problem that estimation range is limited. The estimation range of the proposed algorithm based on evolutionary game reaches the upper bound, and MSE of estimation is free from the influence of frequency offset, that is, the proposed algorithm is superior to other algorithms with more robust convergence and applicability.

Because evolutionary game optimization is heuristic algorithm, closed form solution of expression can't be obtained in solving process, the complexity is higher than EM algorithm. But high accuracy and large estimation range distinct this algorithm can be used in real environment, especially in off-line optimum strategy analysis.

\section{Conclusion}

Multi-objective optimization has made many significant achievements expanded the scope of application in engineering technology, economic, administration, military and systems engineering. The problem of multiple frequency offsets estimation in a Distributed MIMO Systems is real-world search and optimization problems which are naturally posed as non-linear programming problems having multiple objectives.

In this paper, combining multi-objective optimization theory and evolutionary game optimization, a large frequency offset estimation algorithm with higher estimation precision has been proposed based on evolutionary game theory. Different frequency offsets are mapped as players in the game theory to transform the frequency offset optimization problem to optimized equilibrium point. The performance of this algorithm overcomes the error floor in MSE performance of frequency offsets, and local optimization problem of EM algorithm. The results also indicate that the propose algorithm avoids the occurrence of the situation that objective function may have multiple local optimum, which guarantees the high performance of optimization and also adaptability of the algorithm.

\section{Appendix A. CRLB of the performance of multi- objective estimates derived}

In Distributed MIMO systems, because the frequency offset estimate is unbiased, so the CRLB of the mean square error of estimation equals to the reciprocal of Fisher information.

$$
\operatorname{var}(\hat{\theta})=E\left\{(\hat{\theta}-\theta)^{2}\right\} \geq \frac{1}{J(\theta)}
$$

where 


$$
J(\theta)=E\left\{\left[\frac{\partial}{\partial \theta} \ln f(x \mid \theta)\right]^{2}\right\}=-E\left\{\frac{\partial^{2}}{\partial \theta^{2}} \ln f(x \mid \theta)\right\}(\text { A.2) }
$$

$$
\operatorname{CRLB}\left(\mathbf{h}_{k}\right)=\frac{\sigma^{2}}{2}\left\{\operatorname{Re}\left(2 \mathbf{U}_{k}^{-1}+\mathbf{U}_{k}^{-1} \mathbf{T}_{k}\left[\operatorname{Re}\left(\mathbf{W}_{K}\right)^{-1}\right] \mathbf{T}_{k}^{H} \mathbf{U}_{k}^{-1}\right\}\right.
$$

$f(x \mid \theta)$ denotes conditional distribution density function

Through the Fisher information matrix, the lower limit of frequency offset estimation can be found, this provides an excellent theoretical basis for measure of the algorithm

Define the following vector:

$$
\boldsymbol{\eta}=\left[\begin{array}{lll}
\operatorname{Re}(\mathbf{h})^{T} & \operatorname{Im}(\mathbf{h})^{T} & \boldsymbol{\varepsilon}^{T}
\end{array}\right]^{T}
$$

where

$$
\begin{aligned}
\mathbf{h} & =\left[\begin{array}{llll}
\mathbf{h}_{1}^{T} & \mathbf{h}_{2}^{T} & \cdots & \mathbf{h}_{M_{R}}^{T}
\end{array}\right]^{T} \\
\boldsymbol{\varepsilon} & =\left[\begin{array}{llll}
\varepsilon_{1} & \varepsilon_{2} & \cdots & \varepsilon_{M_{T}}
\end{array}\right]^{T}
\end{aligned}
$$

Estimated vector $\boldsymbol{\eta}$ 's Fischer information matrix $F$ is a diagonal matrix

$$
\mathbf{F}=\left[\begin{array}{llll}
\mathbf{F}_{1} & & & \\
& \mathbf{F}_{2} & & \\
& & \ddots & \\
& & & \mathbf{F}_{m}
\end{array}\right]
$$

where $\mathbf{F}_{k}$ denote the Fischer information matrix of estimating parameter $\boldsymbol{\eta}_{k}$

$$
\mathbf{F}_{k}=\frac{2}{\sigma^{2}}\left[\begin{array}{ccc}
\operatorname{Re}\left(\mathbf{U}_{k}\right) & -\operatorname{Im}\left(\mathbf{U}_{k}\right) & -\operatorname{Im}\left(\mathbf{T}_{k}\right) \\
\operatorname{Im}\left(\mathbf{U}_{k}\right) & \operatorname{Re}\left(\mathbf{U}_{k}\right) & \operatorname{Re}\left(\mathbf{T}_{k}\right) \\
-\operatorname{Im}\left(\mathbf{T}_{k}\right) & \operatorname{Re}\left(\mathbf{T}_{k}\right) & \operatorname{Re}\left(\mathbf{V}_{k}\right)
\end{array}\right] \text { (A.7) }
$$

where

$$
\left\{\begin{array}{l}
\mathbf{U}_{k}=\mathbf{S}_{\varepsilon_{k}}^{H} \mathbf{S}_{\varepsilon_{k}} \\
\mathbf{T}_{k}=\mathbf{S}_{\varepsilon_{k}}^{H} \mathbf{D}_{t} \mathbf{S}_{\varepsilon_{k}} \mathbf{D}\left(\mathbf{h}_{k}\right) \\
\mathbf{V}_{k}=\mathbf{D}^{H}\left(\mathbf{h}_{k}\right) \mathbf{S}_{\varepsilon_{k}}^{H} \mathbf{D}_{t}^{2} \mathbf{S}_{\varepsilon_{k}} \mathbf{D}\left(\mathbf{h}_{k}\right) \\
\mathbf{D}\left(\mathbf{h}_{k}\right)=\operatorname{diag}\left(\mathbf{h}_{k}\right) \\
\mathbf{D}_{t}=\operatorname{diag}(1,2, \ldots N)
\end{array}\right.
$$

Available Frequency estimation's CRLB is

$$
\operatorname{CRLB}\left(\boldsymbol{\varepsilon}_{k}\right)=\frac{\sigma^{2}}{2}\left\{\operatorname{Re}\left(\mathbf{V}_{k}-\mathbf{T}_{k}^{H} \mathbf{U}_{k}^{-1} \mathbf{T}_{k}\right)\right\}^{-1}(\text { A.9) }
$$

At the same time, obtained lower bound of channel estimation's mean square error

$$
\mathbf{W}_{K}=\mathbf{V}_{k}-\mathbf{T}_{k}^{H} \mathbf{U}_{k}^{-1} \mathbf{T}_{k}
$$

As the the frequency synchronization (frequency offset estimation and compensation) of synchronization problems is manly discussed in this paper in, it no longer to discuss the channel estimation.

\section{Appendix B.Quasi-optimal multi-parameter optimization of frequency offset}

Refs.6's method is the first multi-frequency offset estimation method is also low complexity, easy practical method for solving multi-frequency offset. Later, a number of multi-objective optimization methods are built on this analysis or use this method to obtain initial value of frequency offset estimated, then, go on the iterative estimation.

Assume that the delay of all transmitting antenna to receiving antenna are the same, but different frequency offset. Algorithm's core idea is to place each antenna with multistage repeat orthogonal sequences and the same deal for multiple sequences at the receiving end, furthermore, diversity of the results can get frequency synchronization performance. Its essence is to transform multi-objective optimization into multiple singleobjective optimization problems.

Firstly, the data sequence is divided into $M$ subsequences, each sequence is Wlash sequence. b's symbol $l$ is

$$
r_{n, b}(l)=\sum_{m=1}^{M_{T}} a_{m}(l) e^{j 2 \pi f_{m, n} l T} h_{m, n, b}+\eta_{n, b}(l) \quad l=1,, L
$$

where, $a_{m}(l)$ denote $l$ th data launched by transmitting antenna $\mathrm{m}, L$ is the length of training sequence, $f_{m, n}$ denote the frequency offset between transmitting antenna and receiving antenna, $T$ is Symbol period, $\left\{h_{m, n, b}\right\}$ denote tapped delay line coefficients, which is the i.i.d. complex Gaussian variables with zero-mean and variance $1, \eta_{n, b}(l)$ denotes Zero mean complex Gaussian noise random variance, and satisfy:
To the receiving antenna $n$, received the sub-sequence 


$$
\begin{aligned}
E\left\{\eta_{n, b}(l)\right. & \left.\left(\eta_{n^{\prime}, b^{\prime}}\left(l^{\prime}\right)\right)^{\mathrm{H}}\right\} \\
= & \sigma^{2} \delta\left(n-n^{\prime}\right) \delta\left(l-l^{\prime}\right) \delta\left(b-b^{\prime}\right)
\end{aligned}
$$

Training sequence

$$
a_{m}(l)(0 \leq l \leq Q)
$$

is Walsh orthogonal sequence, assume $Q$ is orthogonal sequence's length.

$$
Q=2^{\left[\log _{2} M_{T}\right]}
$$

which mean $\left[\log _{2} M_{T}\right]$ not less than $\log _{2} M_{T}$ ' integer, and satisfy:

$$
\sum_{l=1}^{Q} a_{m}(l) a_{m^{\prime}}(l)= \begin{cases}Q & m=m^{\prime} \\ 0 & m \neq m^{\prime}\end{cases}
$$

The receiving signals is correlated with the local $m$ th training sequences :

$$
\begin{aligned}
R_{a_{m}, 1, b}(k)= & \sum_{p=1}^{P} a_{m}(k P+p) r_{1, b}(k P+p) \\
= & h_{m, 1, b} \sum_{p=1}^{P} a_{m}^{2}(k P+p) e^{j 2 \pi f_{m, n}(k P+p) T} \\
& +\sum_{\substack{m=1 \\
m \neq m^{\prime}}}^{M} \sum_{p=1}^{P} h_{m^{\prime}, 1, b} a_{m^{\prime}}(k P+p) a_{m}(k P+p) e^{j 2 \pi f_{m^{\prime}, 1}(k P+p) T} \\
& +\sum_{p=1}^{P} \eta_{1, b}(k P+p) a_{m}(k P+p) \\
& =E_{m, 1, b}(k)+I_{m, 1, b}(k)+N_{m, 1, b}(k)
\end{aligned}
$$

where, $P$ is correlation length。

Make one difference related, differential distance is $i$, so

$$
\begin{aligned}
C_{m, 1, b}(i) & =E\left\{R_{a_{m}, 1, b}(k)\left(R_{a_{m}, 1, b}(k-i)\right)^{*}\right\} \\
= & \left|h_{m, 1, b} A_{m, 1}\right|^{2} e^{j 2 \pi i f_{m, P} P}
\end{aligned}
$$

When differential distance is set to 1 , then

$$
\begin{aligned}
\hat{C}_{m, 1, b} & (1) \\
& =\frac{1}{\lfloor L / P\rfloor-1} \sum_{k=1}^{\lfloor L / P\rfloor-1} R a_{m, 1, b}(k) R a_{m, 1, b}^{*}(k-1)
\end{aligned}
$$

then

$$
\hat{\varepsilon}_{m, 1}=\frac{1}{2 \pi P T} \arg \left(\hat{C}_{m, 1, b}(1)\right)
$$

Eq. (B.9) shows that Yao's method is actually a multiple single objective optimization method, it ignores the interference caused by multiple objectives, therefore, it cannot gain the best estimation performance, it is a quasi-optimal multi-parameter optimization method.

\section{Acknowledgment}

This work was supported by the National Science Foundation of China under Grant number 71001075, 60602009 and 61032002, “863" Project under Grant No.2008AA04A107 and No.2009AA01Z236.

\section{References}

1. J. Ye, X. Liu and L. Han, Evolutionary game algorithm for continuous parameter optimization. Information Processing Letters, 2004, 91(5):211-219.

2. Yanbing L., Jun H., A Novel Fast Multi -objective Evolutionary Algorithm for QoS Multicast Routing in MANET, International Journal of Computational Intelligence Systems, 2009, v2-3: 288-297.

3. J. Du, X. Lei and S. Li, A Novel Frequency Offset Estimation Method for Distributed MIMO Systems with Large Estimation Range, The Fourth International Conference on Rough Set and Knowledge, Technology(RSKT), 2009.3:1012-1015.

4. Marcus V., Nadia N. and Luiza de Macedo Mourelle, Optimal IP Assignment for Efficient NoC-based System Implementation using NSGA-II and MicroGA, International Journal of Computational Intelligence Systems, 2009, v2: 115-123.

5. Cengiz K., Orhan E. and Mustafa K., A New Artificial Immune System Algorithm for Multi-objective Fuzzy Flow Shop Problems, International Journal of Computational Intelligence Systems, 2009, v2-3: 236-247.

6. Y. Yao and T. Ng. Correlation-based frequency offset estimation in MIMO system. in Proc. IEEE Veh. Technol. Conf., 2003 Fall, 1: 438-442.

7. T. Pham; Nallanathan, A. and Ying-Chang L. Joint channel and frequency offset estimation in distributed MIMO flat-fading channels, IEEE Trans. Wireless Commun., Feb. 2008, 7(2): 648- 656

8. S. A. Rustako, R. Roman. Distributed Antennas for Indoor Radio Communication. IEEE Trans. Commun, Dec. 1987, 35(12):1245-1251.

9. J. Chen, Y. Wu, S. C. Chan and Tung-Sang Ng. Joint maximum-likelihood $\mathrm{CFO}$ and channel estimation for OFDMA uplink using importance sampling. IEEE Trans. on Vehicular Technology, Nov. 2008, 57(6):3462-3470

10. J. Chen, Y. Wu, S. Ma and Tung-Sang Ng. Joint CFO and channel estimation for multiuser MIMO-OFDM 
systems with optimal training sequences. IEEE Trans. on Signal Processing, Aug 2008, 56(8):4008-4019.

11. Brad W. Zarikoff and James K. Cavers. Multiple Frequency Offset Estimation for the Downlink of Coordinated MIMO Systems. IEEE J. on Selected Areas of Commun. , Aug. 2008, 26: 901-912.

12. M. Morelli and U. Mengali. Carrier-frequency estimation for space-diversity reception over selective channels. IEEE Proc. Commun., Feb.2002, 149:58-64.

13. P. Dempster, N. M. Laird, and D. B. Rubin. Maximum likelihood from incomplete data via EM algorithm. J. Royal Stat. Soc. Series B, Oct. 1993, 39(1): 447-450.

14. T. K. Moon. The expectation-maximization algorithm. IEEE Signal Processing Mag., Nov. 1996, 13:47-60.

15. G. J. McLachlan and T. Krishnan. the EM Algorithm and Extension. New York: Wiley, 1997.

16. X. L. Meng and D. B. Rubin. Maximum likelihood estimation via the ECM algorithm: A general framework. Biometrika, June 1993, 80(2): 267-278.

17. T. A. Fesler and A. O. Hero. Space-alternating generalized expectation maximization algorithm. IEEE Trans. Signal Processing, Oct. 1994, 42(10):2664-2677.

18. J.F. Nash. Non-cooperative games. Ph.D. thesis, Mathematics Department, Princeton University, 1950 